
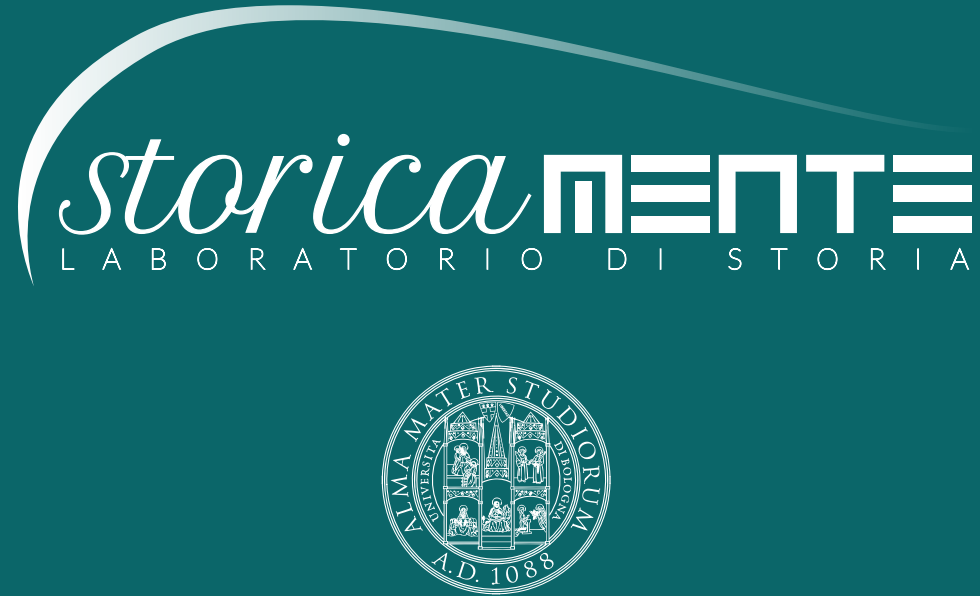

ALMA MATER STUDIORUM

Università di Bologna

Dipartimento di Storia Culture Civiltà

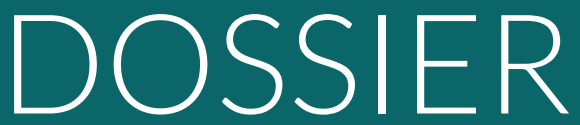

LA POLITICA OLTRE L'IMPERATORE: STRUTTURE, STRATEGIE ED EVOLUZIONE DEI POTERI DIETRO IL TRONO A ROMA E A BISANZIO 


\section{STORICAMENTE.ORG}

\section{Laboratorio di Storia}

Marco Fasolio

Dentro il sistema e contro l'impero: Teodoro Gabras e la costruzione dell'autonomia pontica dopo la battaglia di Manzicerta

Numero $14-2018$

ISSN: 1825-411X

Art. 29

pp. 1-36

DOI: $10.12977 /$ stor719

Editore: BraDypUS

Data di pubblicazione: 19/12/2018

Dossier: La politica oltre l'imperatore: strutture, strategie ed evoluzione dei poteri dietro il trono a Roma e a Bisanzio 


\title{
Dentro il sistema e contro l'impero: Teodoro Gabras e la costruzione dell'autonomia pontica dopo la battaglia di Manzicerta
}

\author{
MARCO FASOLIO
}

Univ. Genova, DAFIST

Scion of an illustrious Anatolian military lineage of probable Armenian descent whose access in the Byzantine aristocracy is datable to the second half of the 10th century, Theodore Gabras was known for being a soldier with formidable abilities. Around 1075, he was able to reconquer Trebizond, the Pontic city which was the capital of Chaldia. At the beginning of the Eighties of the 11th century, he was nominated duke of Chaldia by Alexios I Komnenos, but, as Anna Komnene states, he immediately behaved as the government given to him was his own personal appanage and he handled it as it was his own private property. His career testifies a historical phase, the second half of the 11th century, in which the Byzantine institutions and political and social textures experienced deep changes. In the disruption of the eastern defensive system after the defeat of Mantzikert (1071), the territorial rooting of the Anatolian houses let some of them earn room for political autonomy, also thanks to the consensus they enjoyed among the local Church and populations. Theodore knew how to use his political and economic influence in Chaldia in order to integrate himself in the new system of government, but that same influence allowed him to build a sort of personal lordship, which the Gabrades held with mixed fortunes until the first half of the 12th century.

\section{II clan Gabras prima di Teodoro}

Prima di addentrarci nella biografia di Teodoro Gabras e analizzare il ruolo che ebbe in seno alle strutture di potere di Bisanzio nella prima 
età comnena è utile premettere alcune informazioni sulla sua famiglia $\mathrm{e}$ sul contesto storico entro il quale operò e visse.

Le ipotesi più plausibili in merito all'etimologia del nome 'Gabras' rimandano all'armeno e alle lingue che, insieme all'arabo, erano parlate ai confini orientali di Bisanzio, vale a dire il persiano, il curdo e il turco. Per ciò che concerne l'armeno la parola 'goŕ' o 'goŕoz' non solo rimanda a 'Gabras' dal un punto di vista fonetico, ma richiama anche i caratteri tipici di molti esponenti della famiglia, poiché significa similmente al greco 'Yaũpos', 'fiero', 'maestoso', 'coraggioso' [Bartikian 1996, 20-22]. Nel mondo arabo, invece, il termine 'kafir' indicava in modo generico un miscredente, dunque nella maggioranza dei casi un Cristiano, e da esso derivano con ogni probabilità il cognome siciliano 'Cuffaro' e i suoi affini in Italia meridionale. Dal persiano 'gabrak', in origine riservato agli Zoroastriani, discendono invece la parola curda 'gevir' o 'gavir', ossia 'armeno' o 'russo', e il turco moderno 'gavur', cioè 'miscredente' o 'cristiano' in terra islamica. Se negli idiomi contemporanei questa terminologia ha assunto un significato perlopiù dispregiativo rispetto a colui al quale è attribuita, in origine era neutrale e spesso segnalava quei guerrieri armeni o cristiani ortodossi in genere che operavano ai confini tra la basileia e i territori sotto il controllo dei signori musulmani in qualità di akritai [Bausani 1965, 970].

Ambedue le congetture etimologiche ci inducono a presumere che $\mathrm{i}$ membri del clan avevano assunto come soprannome l'aggettivo con il quale i loro compatrioti o i vicini musulmani li designavano e nel corso delle generazioni questo era divenuto ereditario sino a trasformarsi nel nome proprio della famiglia. Se accettiamo questa ricostruzione e osserviamo l'area in cui sono distribuite le attività della maggior parte

Con il termine akritai le fonti bizantine si riferivano perlopiù a quel tipo di guerrieri/signorotti locali le cui attività si svolgevano sulla frontiera tra l'Impero e il dār al-Islām. Sul tema: Makrypoulias 2007. 
degli appartenenti al lignaggio, ossia la Chaldia e i themata limitrofin', siamo propensi a supporre sia che nei Gabras scorresse sangue armeno, forse di quel ceppo rimasto fedele ai dettami del concilio di Calcedonia e dunque correligionario dei Romei [Bartikian 1996, 24-25], sia che i loro membri appartenessero a quella folta schiera di lignaggi guerrieri del Ponto interno superficialmente o per nulla ellenizzati descritta da Costantino VII (912-959) in un passo del De thematibus ${ }^{3}$. Possiamo dunque considerare i Gabras una stirpe 'tipicamente pontica' e, tenuto anche conto della presunta origine del loro nome, verosimilmente di radicate tradizioni militari. È facile immaginare, allora, che a partire all'incirca dal IX-X secolo avessero accumulato un certo patrimonio fondiario nelle zone di loro pertinenza, al fine di sostenere l'attività bellica propria degli akritai e, pertanto, nutrissero un profondo e radicato interesse nel controllo politico ed economico del territorio, in modo analogo agli arconti della Chaldia loro pari.

Il primo Gabras di cui esistano tracce nelle fonti presenta nel carattere, o meglio nel caratteraccio, molti dei tratti che in seguito avrebbero contraddistinto numerosi tra gli esponenti della famiglia. Costantino Gabras appare per la prima volta nella Storia di Scilize nel 977/978 alla guida dell'ala destra dell'esercito di Barda Sclero, insieme agli alleati armeni e musulmani dell'usurpatore, che a Rhegeas sconfisse le truppe lealiste guidate da Leone Protovestiario ed Eustazio Maleinos. Forse Costantino conobbe Barda Sclero mentre questi era il governatore della Chaldia ${ }^{5}$ prima dello scoppio della guerra civile e vide in lui un campione delle aspirazioni e dei propri interessi, del proprio ceto sociale e della propria terra potenzialmente migliore del basileus, perciò decise di abbracciarne la causa. Da buon Pontico, abituato a convivere con la

Cioè Teodosiopoli, Colonea, Neocesarea e Charsianon: Bryer 1980, 166-168.

Costantino Porfirogenito 1952, 73.

Ioannes Scylitzes 1973, 321-322.

Yahyāa al-Anțakī 1998, 158-159; Kratchovski, Vasiliev 1936, 372. 
popolazione multietnica della sua regione e conscio delle opportunità che si presentavano a un ribelle che avesse avviato la sua impresa in Chaldia ${ }^{6}$, non dovette sicuramente impressionarsi per la composizione dell'armata di Sclero, né tantomeno avvertire disagio nel lottare fianco a fianco con Armeni e Musulmani. Costantino partecipò anche alla prima battaglia di Pankalia, quando nel 978/979 Sclero sconfisse l'armata che Basilio II (976-1025) aveva affidato a Barda Foca, ma nonostante la vittoria dei suoi rimase sul campo. Secondo Scilize egli era «eccessivamente ambizioso» e, forse nella speranza che un suo atto di valore gli avrebbe assicurato un posto di rilievo una volta che Barda Sclero si fosse impadronito di Costantinopoli, ciò lo indusse a contesa ormai terminata a inseguire personalmente il generale avversario in fuga per catturarlo o ucciderlo. Foca, tuttavia, accortosi dell'inseguitore, ne respinse l'assalto e lo uccise?

L'episodio che vide protagonista il secondo membro del clan in ordine cronologico si colloca in un ambito geografico insolito per i primi Gabras, che preferivano muoversi nel più conosciuto e favorevole ambiente anatolico. Giovanni Scilize scrive che tra il 1018 e il 1019 a Tessalonica un tal Gabras, patrizio e arconte del quale omette il nome, si era adoperato per fomentare insieme a Elemagos, anch'egli patrizio e arconte e quasi certamente identificabile con il governatore di Berat Elinagos Sfranze, ultimo signore bulgaro a sottomettersi a Basilio II [Prokić 1906, 35, 48], gli ultimi focolai della resistenza bulgara antibizantina. Il complotto fu subito scoperto e mentre Elemagos riuscì a discolparsi, per poi essere perdonato e reintegrato nei suoi titoli e nei suoi possedimenti, Gabras fu catturato mentre fuggiva verso le sue

La Chaldia era un'eccellente base di partenza per chi volesse ribellarsi al governo centrale, dal momento che era difficilmente raggiungibile dalla capitale via terra ed era ben protetta a sud dalle Alpi Pontiche: Bryer, Winfield 1985, I, 299-300.

La traduzione tra apici è nostra: Ioannes Scylitzes 1973, 325. Su Costantino anche: Bryer 1980, 174, n. 1. 
terre e contestualmente accecato dagli uomini del basileus'. L'appartenenza al clan bizantino dei Gabras di questo individuo non può essere ricostruita con assoluta certezza, sia in quanto la menzione di Scilize è l'unica che lo riguardi, sia poiché il contesto in cui si svolse il tentativo di ribellione era fuori dal tradizionale raggio d'azione degli esponenti del lignaggio. In passato Gyula Moravcsik [1984, II, 108] ipotizzò che si trattasse di un nobile bulgaro legato a Elemagos e che il suo nome coincidesse per puro caso con quello dei Gabras bizantini ${ }^{9}$, ma più di recente Anthony Bryer ha confutato le idee dello studioso magiaro per confermare che anche costui era un membro del lignaggio pontico. Il ragionamento di Bryer è semplice: in primis non sono documentate famiglie bulgare con quel nome, in secundis, dal momento che i primi Gabras erano incorreggibili ribelli e anche costui era un ribelle, è molto probabile che egli fosse un Gabras originario della Chaldia. Non gioca a sfavore di quest'ultima ipotesi che questi abbia sostenuto le velleità di un dinasta bulgaro in Europa, anzi, il suo atteggiamento 'donchisciottesco' nell'abbracciare una causa persa pur di procurarsi l'occasione di ribellarsi al potere centrale era tipico dei primi esponenti conosciuti del casato [Bryer, Fassoulakis, Nicol 1980, 38-39, n. 2]. Per consolidare l'intuizione di Bryer aggiungiamo un particolare che a suo tempo forse egli ritenne secondario. Scilize scrive che Gabras fu catturato «mentre cercava di fuggire verso le sue terre» ${ }^{10} \mathrm{e}$, forse, dietro l'affermazione dello storico si cela l'intenzione di segnalare che allora il ribelle non si trovava nella sua terra natia o in prossimità dei suoi possedimenti fondiari e che, per sfuggire alla giustizia imperiale, aveva tentato di ritornarvi. Non esiste modo di individuare l'ubicazione delle presunte 'terre' di Gabras, ma se pensiamo che il suo tentativo di fuga avesse come obiettivo una località sicura e lontana dalle immediate possibilità di rappresaglia

8 Ioannes Scylitzes 1973, 364.

9 Come lui anche: Mutafciev 1932, 205.

${ }_{10}$ Il corsivo e la traduzione sono nostri: Ioannes Scylitzes 1973, 364. 
dell'imperatore, forse l'Anatolia sarebbe stata una destinazione valida e, a maggior ragione, anche la Chaldia vista la scarsa agibilità dei percorsi terrestri che vi conducevano da Occidente.

Una ventina di anni orsono Hratch Bartikian ha riconsiderato la questione dell'identità di Gabras alla luce della Storia universale dell'armeno Stefano Asolik di Taron, nella quale si legge che i fratelli Cometòpuli erano armeni di Derzene, e Basilio II li aveva chiamati nel 1014 per combattere contro i Bulgari, ma non appena giunti in Europa avevano colto l'occasione per passare al nemico, il quale aveva loro tributato i massimi onori". Bartikian pensa che 'Sfranze' sia una corruzione greca dell'armeno 'Bratzes' e che perciò Elemagos e Gabras, entrambi armeni o di origine armena, altri non fossero se non due tra gli uomini al seguito dei Cometòpuli [Bartikian 1996, 22-24]. Una deduzione verosimile, aggiungiamo noi, se pensiamo alla vicinanza di Derzene alla Chaldia e a come fossero sovente associate nell'amministrazione

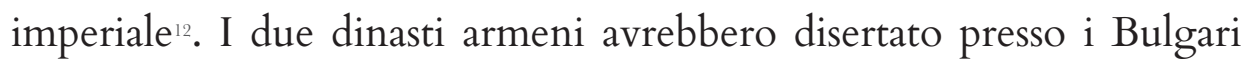
proprio come i Cometòpuli, sarebbero poi ritornati alla fedeltà imperiale e infine l'avrebbero rinnegata una seconda volta nel 1019. Qualora il ragionamento di Bartikian fosse valido confermerebbe quanto scritto da Bryer in merito alla provenienza anatolica dei Gabras, in quanto eliminerebbe i problemi legati alla geografia della ribellione e alla strana alleanza pontico-bulgara. Eppure, ove credessimo alle parole di Stefano di Taron in merito ai Cometòpuli, l'origine armena di Elemagos rimarrebbe una mera congettura e se anche la accettassimo non dimostrerebbe in alcun modo il fatto che egli si trovasse insieme ai fratelli quando costoro avevano risposto all'appello del basileus. Bartikian, inoltre, ignora volutamente e senza fornire spiegazioni la

\footnotetext{
11 Étienne Asołik de Tarôn 1917, 124-125.

12 Si osservino per esempio i sigilli databili a cavaliere tra la prima e la seconda metà dell'XI secolo di Giorgio Drosos, che lo descrivono come 'giudice dell'ippodromo di Chaldia e Derzene' e 'stratego di Chaldia e Derzene': Schlumberger 1894, n. 105; Likhachyov 1911, 20, t. VI, n. 14.
} 
perentoria definizione di 'bulgaro' che Scilize attribuisce a Elemagos ${ }^{13} \mathrm{e}$ compie senz'altro un errore di valutazione quando dichiara che sarebbe stato impossibile che il romeo-armeno Gabras collaborasse all'impresa di un Bulgaro, come avrebbe potuto essere Elemagos, giacché ignora gli esempi dell'arconte di Durazzo Giovanni Criselio e di Ashot Taronita al servizio dello czar Samuele ${ }^{14}$. Salvo questi rilievi, l'impianto del sillogismo di Bartikian resta comunque abbastanza solido e, se ponessimo le sue deduzioni sui Cometòpuli ed Elemagos in una formula più dubitativa, potremmo tenerne conto a sostegno dell'appartenenza alla famiglia pontica di Gabras.

L'ultimo esponente del lignaggio di cui si possano ricostruire le vicende prima del 1071 è Michele ${ }^{15}$. Nel 1040 a Mesanacta, nei pressi di Amorio in Anatolia centrale, il patrizio Gregorio Taronita insieme a Michele Gabras e Teodoro Mesanicte aveva istigato una sollevazione contro il fratello dell'imperatore Michele IV Paflagone (1034-1041), il gran domestico Costantino, ma il tentativo non ebbe successo. Michele e Teodoro furono catturati e accecati, mentre Gregorio, a cui fu risparmiata la vista, fu condotto al cospetto dell'eunuco Giovanni Orfanotrofo, anch'egli fratello del basileus ${ }^{16}$. Visti i precedenti di Costantino Gabras e del Gabras attivo a Tessalonica, non sorprende che Michele avesse aderito a una ribellione e ancora meno che i suoi compagni d'arme fos-

\footnotetext{
13 Ioannes Scylitzes 1973, 364.

14 Ibidem, 342-343, 349. Su Giovanni Criselio anche: Pirivatrič 1997, 78, 106, 110111, 114, 179-193.

15 Probilmente non si tratta dell'ultimo Gabras di cui si conosca l'esistenza negli anni antecedenti alla battaglia di Manzicerta. Tre sigilli databili all'incirca all'XI secolo riportano i nomi di Leone Gabras, spatarocandidato imperiale e chiliarco: Bryer, Dunn, Nesbitt 2003, 63, n. 5; di Marino Gabras: Koltsida-Makri 1996, n. 290; e del sebasto Giovanni Gabras: Seibt, Zarnitz 1997, n. 1.2.8. Molto dubbia è l'attribuzione alla famiglia Gabras di un sigillo appartenente al protospatario e stratego di Cefalonia Catacalone, custodito nella collezione di Dumbarton Oaks: Oikonomides, Nesbitt, Mc Geer 1991-2009, II, n. 1.14.
}

16 Ioannes Scylitzes 1973, 412. 
sero un Taronita e un Mesanicte. La famiglia del primo era da tempo profondamente coinvolta nelle vicende del Ponto e già si era trovata con Romano a fianco di Costantino Gabras tra le schiere in favore di Barda Sclero $^{17}$ [Adontz 1936, 21-23], mentre un parente del secondo aveva figurato tra i principali fautori dell'insurrezione di Barda Foca ${ }^{18}$. La storia familiare dei tre insorti, insomma, rendeva assai probabile che trovassero un accordo per avviare una sollevazione contro il basileus. Il comportamento di Michele, terzo membro della famiglia menzionato in fonti letterarie e terzo ad essersi ribellato a Costantinopoli, corrobora la vocazione del clan a mal tollerare la pace con il governo centrale e ad aderire con convinzione alle iniziative di coloro che vi si opponevano, qualunque fosse la ragione alla base della sollevazione, chiunque ne fosse il promotore e qualunque fosse il seguito di quest'ultimo. In aggiunta, la tipologia delle famiglie coinvolte nella sedizione, segnala che nel momento in cui i Gabras emersero dall'anonimato della classe arcontale pontica tra la fine del X secolo e l'inizio dell'XI, scelsero di vincolare i propri destini a un ristretto nucleo di schiatte militari anatoliche dal consolidato 'pedigree ribelle' e puntarono decisamente su questa rete per la loro ascesa nell'aristocrazia microasiatica, piuttosto che sul servizio all'impero e il prestigio e gli onori che avrebbero potuto derivarne.

\section{II contesto storico}

La parabola di Teodoro Gabras, il più celebre tra i membri medievali del suo lignaggio, si compie nel giro di poco più di un trentennio, tra il 1067 e il 1098, in un periodo nel quale la basileia aveva esperito profon-

Ioannes Scylitzes 1973, 320.

18 Partita come quella di Barda Sclero poco tempo dopo la sua nomina a duca di Chaldia: Ibidem, 291-294; Leo Diaconus Caloënsis 1828, 95-96; Ioannes Zonaras 1841-1897, III, 525. 
di cambiamenti nel sistema politico e nella geografia dei suoi domìni e delle relazioni internazionali. Dopo oltre mezzo secolo di continue lotte per il trono, cominciate alla morte di Costantino VIII nel 1028, nel 1081 con Alessio I si era installata a Costantinopoli la dinastia dei Comneni, che si sarebbe ininterrottamente mantenuta sul trono sino al 1185 e avrebbe modificato in profondità i connotati degli antichi assetti di potere bizantini. A Occidente l'avvento dei cavalieri normanni in Italia meridionale erose rapidamente quanto era rimasto dei possedimenti imperiali, fino a espellere i Romei dalla penisola in seguito alla caduta di Bari nel 1071. A Oriente dell'impero, ossia nell'area alla quale erano maggiormente legati i destini dei Gabras, a partire dalla seconda metà degli anni Quaranta dell'XI secolo si era affacciata la minaccia dei Turchi. Dal regno di Costantino IX Monomaco in avanti tutti i basileis furono in qualche modo costretti ad affrontare in modo diretto le tribù provenienti dalle steppe dell'Asia centrale. Con l'incorporazione dei principati armeni e georgiani che sino a quegli anni avevano funzionato da cuscinetto tra l'Anatolia bizantina e i potentati islamici dipendenti dal califfato di Bagdad che con essa confinavano, l'impero si trovò alla mercé delle scorrerie turche [Holmes 2005, 303-313]. Il sistema difensivo creato da Basilio II era incardinato sulla signoria armena di Ani, dove regnavano i principi georgiani della casa bagratide, ma quando nel 1045 Costantino IX decise di esautorare Gagik II e di inglobare la regione nell'impero per farne un tema, le frontiere di Bisanzio coincisero dapprima con quelle del califfo e degli emiri suoi clienti, poi, in seguito alla nomina di Toghrul beg (1037-1063) a sultano, con quelle del ben più bellicoso sovrano selgiuchide [Angold 1997, 35-55; Vryonis jr. 1971, 1-68].

Oltre ai pericoli che minacciavano la basileia dall'esterno, il generale clima di incertezza era acuito da una profonda crisi delle istituzioni e del prestigio imperiale, che si traduceva nello sfibrarsi dell'amministrazione statale il cui sistema, imperniato sui temi, iniziava chiaramente a mostrare i segni del tempo. Parallelamente la crisi si sostanziava in una 
preoccupante perdita della capacità da parte della corona di coagulare le forze dei maggiori casati aristocratici. L'erosione della base imponibile dovuta alla progressiva abolizione dei vincoli che impedivano ai magnati di acquisire le proprietà dei contadini-proprietari e l'uso spesso scellerato del tesoro imperiale da parte degli epigoni del Bulgaroctono depauperarono le casse dello stato e ciò costrinse i basileis a incrementare le imposizioni fondiarie e a procedere con la fiscalizzazione dei servizi di strateia. In questo modo la struttura e la coesione sociale dei choria furono ulteriormente indebolite, mentre al latifondo era consentito di penetrare in maniera vieppiù pervasiva nella campagna bizantina, alterandone i connotati. In aggiunta la trasformazione dei tradizionali obblighi militari degli stratiotai in imposte sulle loro terre provocò, come scrive a chiare lettere anche Michele Attaliate, un drastico calo negli effettivi a disposizione dell'esercito e obbligò i basileis dei quarti centrali dell'XI secolo a un ricorso sempre più massiccio ai mercenari ${ }^{19}$. L'abrogazione delle leggi antimagnatizie permise all'aristocrazia fondiaria di ampliare sensibilmente le proprie ricchezze e di conseguenza la presa sui territori e le comunità in cui i suoi esponenti erano radicati e questo produsse una crescita economica non trascurabile, in particolare sotto Costantino IX, allorché il basileus svalutò il nomisma per far fronte all'incremento nel volume degli scambi monetari [Laiou, Morrisson 2007, 90-165; Hendy 1985, 35-137; Laiou 2002, 736-770; Morrisson 1976, 3-48]. L'ascesa dei lignaggi anatolici, coincise, tuttavia, con un'inesorabile allentarsi del controllo sulle province da parte dell'instabile governo centrale. La prolungata incapacità dell'impero nel coinvolgere gli esponenti più validi dell'aristocrazia nella gestione dell'apparato pubblico era ascrivibile in misura significativa alle continue lotte per il potere supremo, che sovente si traducevano nell'elevazione al trono di personaggi drammaticamente inadeguati ad affrontare le trasformazio-

19 Miguel Ataliates 2002, 58-59. Sull'esercito si veda anche il recente: Haldon 2017, 581-593. 
ni in corso nella basileia. Se le dignità palatine e il servizio nei ranghi dello stato non rappresentavano più uno sbocco appetibile per le élite, l'alternativa per i membri dei lignaggi militari dell'Anatolia consisteva nello sfruttare l'evidente indebolimento delle facoltà di governo sulle periferie da parte dei funzionari inviati da Costantinopoli, per accrescere il proprio peso e la propria influenza a livello locale, ritagliandosi così spazi di autonomia politica ed economica impensabili sotto la dinastia basilide [Neville 2004, 5-118; Angold 1997, 81-98; Oikonomides 1976, 125-152; Cheynet 1983, 453-477; Glykatzi-Ahrweiler 1976, 99-124, Andriollo, Métivier 2017, 505-530] ${ }^{20}$.

Alla vigilia della battaglia di Manzicerta del 1071, da un punto di vista esterno l'impero era in apparente buona salute, poiché i suoi confini erano stati allargati rispetto a quelli ereditati da Basilio II e nulla sembrava far presagire l'imminente disastro, e tuttavia era nelle sue strutture interne che Bisanzio si trovava sull'orlo del collasso: bastò una sconfitta, peraltro di modesta entità e con un numero relativamente basso di caduti [Cheynet 1980, 410-418], perché il castello di carte su cui si reggeva crollasse in un solo colpo. La disaffezione dell'aristocrazia nei confronti del trono la rese indisponibile a collaborare attivamente alla difesa del territorio, già privato dei necessari presìdi militari a causa del deciso decremento della forza numerica dell'esercito, e aprì ai Turchi le porte dell'Anatolia. Il caos e la disorganizzazione che regnavano a Costantinopoli e nei temi periferici facilitarono la trasformazione di quella che avrebbe dovuto essere un'incursione temporanea in un'occupazione permanente e nel giro di dieci anni l'assenza di qualsivoglia coordinamento centrale nella resistenza all'invasione consentì ai Selgiuchidi, non di rado affiancati dai Romei ai quali era più inviso il governo centrale, di giungere sino alle sponde dell'Egeo [Vryonis jr. 1971, 69-113, Cheynet 1998, 131-147].

\footnotetext{
20 Per un inquadramento sull'aristocrazia e il suo ruolo nell'amministrazione provinciale, in particolare per ciò che concerne i secoli IX-XI, si veda anche l'eccellente monografia di Luisa Andriollo: Andriollo 2017.
} 
Lo sfarinamento dell'apparato statale non si palesò soltanto in seguito al crollo improvviso delle difese in Anatolia, ma anche con il fiorire, a cavaliere degli anni Settanta dell'XI secolo, di una serie di signorie personali interne alla basileia imperniate perlopiù sui themata orientali. La prima in ordine cronologico fu quella di Roberto Crispino, un mercenario normanno, a detta di Attaliate proveniente dall'Italia, al seguito dell'esercito di Romano IV Diogene (1068-1071). Durante la campagna antiturca del basileus del 1068, Crispino sfruttò le debolezze dell'amministrazione provinciale e con il pretesto di un ritardo nei pagamenti si staccò dal corpo principale dell'esercito per asserragliarsi nel 1069 con i suoi uomini nella fortezza di Maurocastro, forse coincidente con la città di Colonea, nel tema Armeniakon. Con l'appoggio dalla popolazione locale, ormai disaffezionata rispetto all'amministrazione imperiale, Crispino iniziò a sequestrare il denaro trasportato dagli esattori delle tasse bizantini nella zona sotto il suo controllo atteggiandosi a signore territoriale. Questo allarmò Romano IV, che tentò per ben due volte di cacciare il ribelle dalla sua roccaforte, ma invano, dal momento che sia il primo ridotto contingente bizantino, sia i cinque tagmata inviati in seguito dal basileus ${ }^{21}$ furono respinti. L'esperienza separatista, tuttavia, si concluse nel giro di un anno, poiché fu lo stesso Crispino a recarsi presso l'imperatore per rinnovare il suo omaggio e domandare il perdono per la sua condotta. Romano IV, però, indignato a causa della precedente infedeltà del suo mercenario, ne rifiutò la sottomissione e lo fece catturare e imprigionare ad Abido ${ }^{22}$.

Secondo Niceforo Briennio Roussel de Bailleul era uno dei seguaci di Crispino $^{23}$ ed è possibile che ne avesse condiviso la ribellione del 1069, ma presumibilmente senza subire la medesima punizione del suo comandante, giacché nel 1071 era al fianco di Giuseppe Tarcaniote

${ }_{21}$ Guidati da Samuele Alusiano.

22 Miguel Ataliates 2002, 93-95.

23 Nicéphore Bryennios 1975, 149. 
nel distaccamento dell'esercito di Romano IV che avrebbe dovuto soccorrere il basileus a Manzicerta ${ }^{24}$. Alla morte di Crispino - nel frattempo riabilitato e reimpiegato da Michele VII Ducas (1071-1078) ${ }^{25}$ - nel 1073 Roussel ne prese il posto alla guida dei mercenari franchi. La sua ribellione ebbe inizio in quello stesso anno, mentre si trovava a capo di un piccolo contingente di Franchi insieme alle truppe sotto il comando del domestico d'Oriente Isacco Comneno e accompagnate dal fratello di questi Alessio, il futuro Alessio I, nel corso di una spedizione antiturca. Quando l'armata giunse a Iconio Roussel ebbe un dissapore con i suoi compagni d'arme e decise di allontanarsi con gli uomini del suo seguito. Negli anni tra il 1073 e il 1075, quando fu definitivamente catturato dai Turchi e da questi ceduto ad Alessio Comneno, il mercenario latino si era impadronito di una fetta cospicua dell'Anatolia bizantina con la Cappadocia, buona parte della Paflagonia, Amasea, che era la 'capitale' dei suoi domìni, e l'Armeniakon e aveva attratto a sé non solo molti dei mercenari franchi al servizio dell'impero, ma anche numerosi Romei ${ }^{26}$. Quella di Roussel fu una signoria territoriale vera e propria e, sebbene sia stata liquida nei confini e di breve durata, ebbe la solidità necessaria per opporsi efficacemente sia ai Bizantini, che rivendicavano la sovranità sui territori occupati, sia ai Turchi, che provavano a scalzare l'intraprendente generale normanno, e per non soccombere in occasione di una sua momentanea cattura. La creatura di Roussel fu di fatto la sola entità politica che nella prima metà degli anni Settanta dell'XI secolo impedì ai Turchi la conquista dell'intera Anatolia bizantina: la

${ }_{24}$ Distaccamento che, tuttavia, non raggiunse mai il campo di battaglia e contribuì alla sconfitta dell'esercito imperiale: Miguel Ataliates 2002,111.

25 Ibidem, 126-127.

26 Persino Giovanni Ducas, fratello di Michele VII, in un primo momento avversario di Roussel, catturato nel corso di un tentativo bizantino di disfarsi del ribelle, una volta liberato fu proclamato imperatore dalle truppe del mercenario franco e per un tratto ne assecondò i progetti: Ibidem, 135-152; Nicéphore Bryennios 1975, 149, $167-$ 173, 177, 185-195; Ioannes Zonaras 1841-1897, III, 709-712. 
facilità con cui era stata costruita dimostra quanto fosse ormai precaria la situazione dell'Anatolia bizantina all'indomani di Manzicerta. Una precarietà che permetteva a chiunque potesse contare su un piccolo esercito e godesse di un certo prestigio personale, di ricavare un principato territoriale all'interno dei confini imperiali e difenderlo sia dai Turchi sia dai Bizantini, purché contasse sul sostegno della popolazione e della classe arcontale locale.

Non troppo dissimile fu la vicenda di Filareto Bracamio, discendente di una stirpe principesca armena che dal X secolo in avanti aveva prestato i suoi servigi all'impero occupando le più cospicue cariche militari ${ }^{27}$ [Cheynet, Vannier 1986, 57-74; Cheynet 2008, II, 377-412]. Nel 1069, durante la campagna che avrebbe condotto al disastro di Manzicerta, Romano IV assegnò a Filareto il comando della maggior parte dell'esercito affinché affrontasse i Turchi, mentre egli si sarebbe ritirato con il resto degli uomini alle pendici del Tauro. Bracamio, tuttavia, subì una pesante sconfitta che comportò ingenti perdite per l'armata bizantina e fu costretto a ripiegare verso gli accampamenti di Romano IV, da dove sarebbe poi ripartito al fianco del basileus per incontrare sul campo di battaglia il sultano Alp Arslan ${ }^{28}$. Sfuggito alla cattura durante la battaglia, ma indisponibile a dialogare con Michele VII a causa del trattamento che aveva riservato all'ex-imperatore, al quale Filareto doveva buona parte della sua ascesa nei ranghi dell'esercito bizantino, insieme a una folta milizia composta in prevalenza da Armeni, ma non priva di soldati romei e di altre etnie, si ribellò al nuovo basileus e costruì un principato personale incardinato sulle imprendibili fortezze della Cilicia e che si estendeva sino alle metropoli siriane di Edessa e Antiochia. Coadiuvato dall'aristocrazia in prevalenza armena delle regioni nelle

Sugli Armeni in servizio nell'Impero durante gli anni di Filareto Bracamio: Cheynet 1996, 67-78.

${ }_{28}$ Miguel Ataliates 2002, 99-100. 
quali si era insediato ${ }^{29}$, seppe resistere efficacemente alla pressione tur$\mathrm{Ca}^{30}$ mantenendo a un tempo piena indipendenza da Costantinopoli. Con la deposizione di Michele VII e l'avvento sul trono di Niceforo III Botoniate (1078-1081) il principale ostacolo per la riconciliazione con Bisanzio era svanito, pertanto Filareto decise di prestare omaggio al nuovo imperatore, il quale, in cambio, sanzionò ufficialmente il potere che de facto deteneva tra la le montagne della Cilicia e la Celesiria. Nonostante l'avallo formale del governo di Costantinopoli, che comunque non poté mai esercitare un effettivo controllo sull'operato di Filareto e dei suoi alleati, né tantomeno fornire un supporto concreto alla difesa del territorio, il dominio di Bracamio fu effimero. Antiochia cadde nel 1084 in mano all'esiguo contingente guidato dall'emiro di Nicea ${ }^{31}$ a causa del tradimento del figlio di Filareto, mentre il resto della signoria si sgretolò rapidamente nel corso delle offensive selgiuchidi degli anni seguenti ${ }^{32}$.

\section{Teodoro Gabras tra storia e folclore}

Teodoro Gabras appare per la prima volta insieme alla moglie Irene ${ }^{33}$ nell'illustrazione dedicatoria di un Tetravangelo del 1067, il Codex Pe-

29 Tra i nobili armeni che sostenevano l'iniziativa di Filareto spiccavano le figure di Gabriele di Melitene e di Basilio Apocape, signore di Edessa.

30 Nel 1083, dopo la riconciliazione con l'Impero, Filareto pensò per un momento di convertirsi all'Islam pur di conservare il potere.

31 Allora era Suleiman ibn-Kutlumus.

32 Tsolakes 1968, 182-184; Nicéphore Bryennios 1975, 203-205; Miguel Ataliates 2002, 215-216; Dostourian 1993, 137-152; Michel le Syrien 1899-1910, III, 173-179. Su Filareto si vedano anche: Yarnley 1972, 331-353; Cheynet 2008, 390-410, n. 13.

33 L'identificazione è possibile grazie alla didascalia al disotto del ritratto della coppia, nella quale è scritto «Dio aiuta la tua serva Irene Gabras» 
tropolitanus Graecus 291 ${ }^{34}$, sotto la quale appare una didascalia che lo descrive come "Teodoro patrizio e topoterete Gabras servo di Dio» [Beneševič 1912-1925, II, 52, t. 37]. Se ci fidiamo di Anna Comnena quando afferma che Teodoro era originario della Chaldia interna ${ }^{35}$, possiamo dedurre che sul finire degli anni Sessanta dell'XI secolo egli era uno stimato membro della classe arcontale pontica, degno dell'axia di patrizio, ma che la sua autorità era strettamente limitata a livello locale, giacché il titolo di 'topoterete' indicava in quegli anni un piccolo signore territoriale, privo di una qualifica o di un incarico formale conferito dall'imperatore [Cheynet 1984, 215-224]. Se ci basassimo sulla cosiddetta Ballata di san Teodoro Gabras di Arta ${ }^{36}$, potremmo precisare l'affermazione della figlia di Alessio I e identificare nel villaggio pontico di Arta il luogo natio di Teodoro. Odisseo Lampsides, tuttavia, ha dimostrato inequivocabilmente che la Ballata è in larga parte un pastiche composto da brani scelti dalla Cronaca di Costantino Manasse e dal Digenis Akritas, le cui informazioni su Teodoro non sono più antiche della fine del XIX secolo [Lampsides 1959, 33-38]. Anche se l'indicazione sulle origini di Teodoro è compatibile con quanto scrive Anna e può aver assorbito qualche tradizione locale, l'indicazione della ballata è una pura suggestione letteraria e come tale va considerata. Attenendoci alla versione fornita dall'Alessiade, in un periodo imprecisato collocabile tra la metà degli anni Settanta e la metà degli anni Ottanta dell'XI secolo Teodoro aveva di sua spontanea iniziativa riconquistato Trebisonda dai Turchi, che presumibilmente l'avevano occupata poco dopo la battaglia di Manzicerta. Nella descrizione di Anna Comnena Teodoro appare al contempo come un uomo energico e violento e un soldato irresistibile,

Anticamente nel Codex Sinaiticus Graecus 172 della biblioteca del monastero di Santa Caterina del Sinai, fu estratto a suo tempo da Porfirio Uspenskij, che lo portò con sé in Russia, dove tuttora è custodito.

35 Anne Comnène 1937-1945, II, 151.

36 Edita dal chierico Giovanni Iosefidis Gabras, un Romeo del Ponto, sul finire del XIX secolo: Iosephides Gabras 1890. 
capace di conseguire qualsiasi obiettivo si proponesse di raggiungere e che, una volta impadronitosi del capoluogo della Chaldia, lo tenne per sé insieme al territorio circostante come se fosse una proprietà privata $^{37}$, difendendolo dalle brame di conquista dei Turchi e dei Georgiani [Bryer 1980, 175, n. 3]. Dopo qualche tempo, ossia tra il 1085 e il 1090 circa, Teodoro si recò a Costantinopoli presso l'imperatore Alessio I, il quale però lo avvertiva come una figura scomoda e pertanto decise di nominarlo duca di Trebisonda, pur di liberarsene. In cambio Teodoro ottenne che suo figlio Gregorio fosse promesso in matrimonio a una figlia ${ }^{38}$ del sebastocratore Isacco Comneno, fratello di Alessio I, a patto che rimanesse in qualità di ostaggio a corte.

Poco dopo il rientro a Trebisonda, Irene, la consorte di Teodoro, morì ed egli decise di risposarsi con una nobildonna georgiana proveniente dall'Alania, cugina da parte di madre della moglie di Isacco Comneno ${ }^{39}$. Il matrimonio di Teodoro, nondimeno, sciolse d'ufficio il fidanzamento tra Gregorio e la figlia del sebastocratore, in virtù dei divieti imposti dal diritto canonico bizantino nei matrimoni tra parenti, parenti spirituali e affini fino al sesto grado [Darrouzes 1977, 112-117]. Giunto a conoscenza dell'accaduto Teodoro reclamò la restituzione del figlio rimasto presso l'imperatore, ma inutilmente: Alessio I non intendeva ottemperare alle richieste del duca, in quanto la custodia di Gregorio costituiva la sola garanzia del basileus contro una eventuale ribellione di suo padre. Quest'ultimo, allora, si recò personalmente a Costantinopoli in visita al figlio nel 1091, intenzionato a rapirlo; tuttavia, dopo avere ingannato le guardie preposte al controllo di Gregorio ed essersi imbarcato con lui su una nave mercantile, fu intercettato da una flottiglia imperiale tra Eginopoli e Karambis in Paflagonia. Teodoro dovette dun-

Anne Comnène 1937-1945, II, 151.

38 Forse Maria: Ibidem, II, 151-152.

39 Ibidem, II, 51-152. Sulla seconda moglie di Teodoro Gabras anche: Vannier 1998, II, 677-679. 
que riconsegnare il figlio al basileus. Lo fece malvolentieri e solo dietro le esplicite minacce di rappresaglia nei suoi confronti, anche se riuscì a strappare la promessa di fornire a Gregorio un'educazione a corte e insieme l'impegno del suo fidanzamento con Maria Comnena, figlia di Alessio I. Anni dopo, intorno al 1094, Gregorio Gabras, insoddisfatto del trattamento ricevuto a corte e da parte del suo precettore, l'eunuco Michele, attendente di Irene Ducas ${ }^{40}$, organizzò in collaborazione con alcuni membri della corte ${ }^{41}$ un piano per sfuggire alla prigionia costantinopolitana e, forse, ricongiungersi con il padre sul Ponto. Tradito da uno dei suoi, però, Gregorio fu scoperto dagli agenti dell'imperatore e dopo avere rivelato quali fossero le sue intenzioni e chi fossero i suoi complici, fu immediatamente spedito a Filippopoli affinché il governatore locale ${ }^{42}$ lo tenesse sotto stretta sorveglianza e gli impedisse ulteriori tentativi di fugat ${ }^{43}$.

Mentre, in seguito alla prigionia in Tracia, Gregorio Gabras svanisce dagli orizzonti degli storici bizantini, Teodoro ricompare nel 1098 nei pressi di Paipert, che egli stesso aveva conquistato poco tempo prima. Ibrahim, il sultano turco ghaznavide del Khorasan [Bosworth 1998, 116], aveva posto il figlio Ismaele alla guida di un esercito affinché affrontasse Alessio I, in procinto di raggiungere Antiochia per sollevarla dall'assedio. Gli imperiali, tuttavia, si erano ritirati a Filomelio e Ismaele decise di cambiare obiettivo, volgendo le truppe a nord, verso l'Anatolia. Informato dell'imminente pericolo Teodoro Gabras anticipò l'arrivo del nemico e lo affrontò fuori dalle mura di Paipert ${ }^{44}$. Qui si

40 L'imperatrice, moglie di Alessio I.

${ }_{41}$ Ossia Giorgio Decano, Eustazio Camitza e il pincerna, un ufficiale di corte, Michele. Sul titolo, derivato dal verbo 'Émıкєрávvvuı', ossia 'mischio il vino', rimandiamo a: Guilland, I, 242-250.

42 Si trattava di Giorgio Mesopotamita.

43 Anne Comnène 1937-1945, II, 151-152.

44 Ibidem, III, 29-30. 
esaurisce il racconto dell'Alessiade su Teodoro e non esistono altre fonti coeve o medievali che forniscano indizi in merito a quale fu l'esito dello scontro. Nondimeno un Sinassario - rinvenuto a Trebisonda da Papadopoulos-Kerameus agli inizi del XX secolo presso un'abitazione privata e contenuto in un volume datato 1884 , ma composto presumibilmente in loco tra il 1765 e il 1768 da un certo Giovanni Oikonomos di Pythiana per soddisfare le esigenze liturgiche della Chiesa trebisontina [Papadopoulos-Kerameus 1906, 133-134] - descrive, rielaborando materiale agiografico di area pontica oggi non più accessibile, la cattura di Gabras nel corso della battaglia di Paipert da parte di un tal Amir Alì, forse lo stesso Ismaele. Questi avrebbe in seguito condotto Teodoro a Teodosiopoli e, dopo avere inutilmente tentato di convertirlo all'Islam, lo avrebbe poi torturato, ucciso e ne avrebbe trasformato il teschio nella sua tazza personale, bruciando il resto del corpo. La testa di Teodoro, divenuto ormai un martire cristiano, sarebbe stata recuperata all'incirca una ventina di anni dopo da parte del nuovo duca di Chaldia Costantino Gabras, probabilmente il figlio o, secondo il Sinassario, nipote del defunto, per conservarla in un reliquiario posto in un monastero a lui dedicato ${ }^{45}$. Quantunque il racconto del Sinassario sia di molto posteriore agli episodi ai quali si riferisce e possa sembrare fantasioso e un po' folcloristico, il dato in merito alla cattura e al martirio di Teodoro è certamente plausibile, dacché già Giovanni Zonara, la cui opera storica fu composta non molto più tardi del $1118^{46}$, lo cita in associazione al figlio Gregorio, sposo di Maria Comnena, presentandolo come "sebasto e martire» ${ }^{47}$. Altrettanto verosimile è il fatto che le spoglie di Teodoro Gabras siano state accolte in un monastero a lui dedicato,

45 Papadopoulos-Kerameus 1906, 135-137.

46 In quanto la Synopsis historiarum si conclude con la morte di Alessio I, avvenuta appunto nel 1118.

47 Ioannes Zonaras 1841-1897, III, 739. Il martirio di Teodoro Gabras era una nozione ormai consolidata nel XIV secolo, come attesta la menzione del metropolita di Trebisonda nella sua collezione dei miracoli di sant'Eugenio: Rosenqvist 1996, 214. 
giacché in una crisobolla del 1364 l'imperatore di Trebisonda Alessio III Comneno (1349-1390) assegnò ai Veneziani il monastero del «santo megalomartire e sebasto Teodoro Gabras» e l'area circostante affinché vi costruissero il proprio serraglio ${ }^{48}$.

Se per un istante accantonassimo il macabro finale dei resti mortali di Teodoro Gabras potremmo individuare nelle sue vicissitudini sul Ponto un'esperienza paragonabile a quella vissuta, grossomodo negli stessi anni, da Filareto Bracamio. Sebbene Teodoro avesse strappato Trebisonda e la Chaldia dalle mani dei Turchi, mentre Filareto, al contrario, si fosse impossessato di territori che allora si trovavano formalmente ancora sotto il controllo della basileia, ambedue le iniziative erano state spontanee e prive di qualsivoglia aiuto o coordinamento da parte delle autorità costantinopolitane. In sostanza i propositi che li spinsero ad agire in autonomia dall'amministrazione centrale coincidevano, dal momento che sia Teodoro sia Filareto costruirono, non possiamo sapere con quale grado di consapevolezza, una signoria territoriale personale geograficamente compatta, le cui modalità di controllo del territorio erano alternative a quelle della basileia. Per ovvie ragioni la formula nella quale si traduceva l'espressione del potere in quelle entità politiche non poteva essere la struttura burocratica piramidale propria della basileia, ma prevedeva un sistema più snello e meno stratificato. Visti il percorso che Bracamio aveva intrapreso nella formazione della sua signoria, ossia la ricerca del consenso al suo disegno da parte dei principali magnati armeni della Cilicia e della Celesiria, e le specificità dell'ambiente pontico ${ }^{49}$ in cui si mosse Gabras, potremmo supporre che

48 Zakythinos 1932, 34. L'esistenza del monastero è confermata anche da un passo del resoconto dell'assedio di Trebisonda del 1222 di Giovanni Lazaropoulos. L'agiografo di sant'Eugentio racconta che l'imperatore di Trebisonda Alessio I Gido si fermò a pregare nella chiesa dedicata al martire Teodoro Gabras: Rosenqvist 1996, 314. Oltre che: Bryer 1980, 175, n. 3; un'utile profilo biografico di Teodoro si trova in: Skoulatos 1980, 295-298, n. 200.

49 Caratterizzato, specialmente nelle aree rurali, dalla presenza di una classe arcontale piuttosto radicata sul territorio e dalla spiccata vocazione militare: Bryer, Winfield 
l'organizzazione di cui i due si erano dotati fosse di tipo reticolare. Al centro vi era il signore territoriale, al quale rispondevano direttamente i gangli periferici, occupati dagli esponenti delle aristocrazie locali, dal clero secolare e dai monasteri che esercitavano un'autorità informale, ancorché sensibile e riconosciuta dalla popolazione, sulle aree in cui erano radicati. Dopo una fase di piena 'indipendenza' entrambi scelsero di riconoscere la supremazia dell'impero, ottenendone in cambio l'investitura ufficiale sui territori nei quali, in concreto, già governavano, senza che ciò comportasse un effettivo cambiamento nella loro condotta, un'intrusione dei funzionari costantinopolitani o un'alterazione nelle loro trame di potere.

Ciò che distinse l'esperimento di Teodoro Gabras da quello di Filareto Bracamio fu senz'altro l'efficacia nel resistere alle pressioni esterne e la solidità dell'impianto di governo. Filareto, tradito in seno alla sua stessa famiglia, vide il suo principato sgretolarsi non appena un piccolo esercito turco ne varcò i confini, mentre la Chaldia era rimasta in mani cristiane anche dopo la morte di Gabras, come testimonia l'invio di Dabateno in veste di duca di Trebisonda da parte di Alessio I nel $1098^{50}$. Forse Bracamio non era stato abbastanza abile nel coagulare attorno a sé le forze locali in direzione della difesa dagli aggressori esterni e al primo colpo inferto al suo prestigio i tenui legami personali che tenevano insieme il suo dominio si erano sciolti. Al contrario Teodoro Gabras beneficiò di un consolidato sistema di alleanze tra le élite guerriere della Chaldia e il vertice amministrativo del tema, in cui egli si era facilmente inserito sia in virtù del retaggio familiare, sia delle qualità personali ricordate da Anna Comnena. Non solo si era posto alla guida della classe arcontale, ma con le sue sostanze personali aveva garantito, secondo quanto scrive Giovanni Lazaropoulos, la prosecuzione del tra-

1985, I, 301-302.

50 Anne Comnène 1937-1945, III, 75. Prima di essere sostituito da Gregorio Taronita nel 1103 . 
dizionale festeggiamento di sant'Eugenio, patrono di Trebisonda e di Paipert, il 24 giugno di ogni anno, analogamente a quanto gli strateghi e i duchi del passato avevano fatto usufruendo dei fondi dell'erario ${ }^{51}$. Fu probabilmente ancora Teodoro insieme ai suoi parenti, tra i quali vi erano forse anche suo figlio Gregorio e Costantino ${ }^{52}$, a promuovere l'edificazione del monastero di san Giorgio a Cheriana, una località posta tra la Chaldia interna e Colonea, che per volere dei fondatori forniva ogni anno un omaggio in natura al monastero di sant'Eugenio di Trebisonda ${ }^{53}$. Inoltre, alla morte della moglie, Gabras si era sposato con una principessa georgiana, forse per normalizzare le relazioni con il potente vicino e neutralizzarne le aspirazioni sulla Chaldia. Quello di Teodoro fu un comportamento del tutto naturale, quasi obbligato per chi ricopriva una posizione come la sua in un contesto nel quale non avrebbe potuto contare sul concreto sostegno da parte della basileia, a maggior ragione se si considera che il matrimonio fu celebrato quando egli già era duca di Trebisonda e pertanto quasi certamente presupponeva una finalità politico-diplomatica. Teodoro conosceva alla perfezione le dinamiche di potere locali e ciò gli aveva consentito dapprima di riunire le classi dirigenti pontiche sotto la sua egida e poi di formare un legame con l'area iberica in modo da proteggere la sua posizione sia dagli attacchi delle tribù turche sia dalle potenziali ingerenze costantinopolitane. Grazie alle sue risorse personali incoraggiò il sorgere di un sentimento di 'unità nazionale' tramite il finanziamento delle tradizionali manifestazioni della religiosità pontica e il sostegno ai monasteri della Chaldia, così da coinvolgere sia il clero sia la popolazione nel suo progetto politico e garantirne il successo a lungo termine.

\footnotetext{
51 Rosenqvist 1996, 214.

${ }^{52}$ Figlio o nipote di Teodoro, fu a sua volta duca di Trebisonda a partire dalla fine degli anni Dieci del XII secolo. Costantino si ribellò all'Impero intorno al 1126 e governò la Chaldia in piena autonomia da Costantinopoli da quel momento in avanti: Skoulatos 1980, 66-68, n. 40; Bryer 1980, 177, n. 5.
}

53 Rosenqvist 1996, 258-260. 
Estrapolato dal contesto della contesa bizantino-selgiuchide per l'Anatolia di fine XI secolo e dai condizionamenti che necessariamente il ruolo di potere nel Ponto esercitò sulle decisioni politiche di Teodoro, il suo profilo offre alcuni spunti di riflessione in merito alle relazioni che intrattenne con Alessio I e, di riflesso, sulla natura dei suoi interessi economici in Chaldia. Afferrare il sottotesto delle parole di Anna Comnena quando scrive "Allora gli eventi si misero male per l'imperatore. Dal momento che Teodoro Gabras risiedeva a Costantinopoli, l'imperatore, siccome ne conosceva la natura violenta ed energica e voleva allontanarlo dalla Città, lo nominò duca di Trebisonda» è forse la chiave per comprendere l'essenza dei rapporti che intercorrevano tra Teodoro e Alessio I. Poco più avanti Anna afferma che Gabras «una volta presa la città di Trebisonda la rivendicò per sé come se fosse una terra sua propria $»^{54}$ e ciò è stato sinora interpretato come un segnale riguardo all'autonomia con la quale Teodoro aveva agito nella fase di conquista [Skoulatos 1980, 295-298, n. 200; Bryer 1980, 175, n. 3]. Questo genere di ermeneutica è senz'altro corretto, tuttavia la frase della Porfirogenita può celare il fatto che nell'operazione di Gabras vi fosse anche una conseguenza concernente gli assetti economici della regione, oltre ai più tangibili risvolti di tipo politico-signorile. Nel sottrarre Trebisonda e le zone circostanti ai Turchi, forse Teodoro si era arrogato una sorta di 'diritto di conquista', impadronendosi in veste di signore territoriale di quelle terre un tempo di proprietà del fisco imperiale, ma sulle quali lo stato aveva perso le sue prerogative demaniali al momento dell'occupazione selgiuchide ${ }^{5}$. Sulla base di questa ipotesi potremmo arricchire di un ulteriore elemento le ragioni per le quali Alessio I si sentisse tanto minacciato dal dinasta pontico. A differenza della stragrande maggioranza dei membri dell'aristocrazia militare, Comneni compresi,

${ }^{54}$ Anne Comnène 1937-1945, II, 151. La traduzione è nostra.

${ }_{55}$ Forse, proprio grazie alla ricchezza così ottenuta Teodoro era in grado di finanziare le manifestazioni religiose e i monasteri della Chaldia. 
che avevano perso quasi tutte le loro ricchezze fondiarie anatoliche a causa dell'avanzata turca, Teodoro si trovava nella felice condizione di non dover dipendere dalle rogai né tantomeno dalle elargizioni di terre imperiali. Se a questo aggiungiamo che il potere e il prestigio di Gabras non promanavano dalle axiai concesse dal basileus, bensì dalle sue proprie iniziative è evidente come ciò facesse di lui un uomo potente, ma difficilmente controllabile. In sostanza, se la causa prima della posizione politica ed economica di dominio acquisita da Teodoro in Chaldia non era riconducibile al sovrano, questo lo rendeva un'anomalia del sistema di potere bizantino - dove l'imperatore rimaneva la sola fons honorum e al contempo il fulcro della redistribuzione delle ricchezze pubbliche - e perciò un potenziale pericolo per Alessio I.

Nel 2009 Paul Magdalino aveva individuato il nodo del rinnovamento delle élite bizantine tra il periodo tardoantico e l'età medievale nel cambiamento della fonte delle loro ricchezze. Mentre il ceto senatorio romano traeva le proprie sostanze principalmente dalle cospicue proprietà terriere ed era dal punto di vista economico quasi del tutto indipendente dall'imperatore, la nuova classe dominante era giunta alla preminenza sociale grazie alla vicinanza al sovrano. Secondo il bizantinista britannico questa condizione aveva prodotto un'aristocrazia perlopiù cortigiana e dedita al servizio del basileus, dal quale dipendeva quasi completamente per il prestigio e il sostentamento, fosse esso sotto forma di pensioni, concessioni di terre o remunerazioni per gli incarichi svolti [Magdalino 2009, 212-232]. Ora, se analizzassimo in quest'ottica la posizione di Teodoro Gabras nei confronti di Alessio I, di certo sarebbe una forzatura ricondurla al modello delle élite bizantine, giacché si avvicina naturalmente ai canoni indicati da Magdalino per la classe senatoria romana. Certo, un ragionamento del genere è in parte una forzatura, dal momento che in età comnena almeno una quota delle ricchezze dell'aristocrazia, specialmente di area balcanica, non era solo il frutto della generosità del basileus. Nonostante ciò, applicare lo schema elaborato da Magdalino al caso di Teodoro ci consente di sottoline- 
are ulteriormente l'indubbia atipicità della sua condizione nella basileia del tardo XI secolo e di come tale condizione rappresentasse un'insidia per Alessio I. Pertanto, allorché l'imperatore propose a Teodoro il matrimonio tra Gregorio e una principessa del clan regnante, si trattò di un tentativo volto a includerlo nel sistema di potere familistico proprio del regime comneno al fine di stabilire su di lui una qualche forma di controllo. Per quanto attiene poi alla nomina a duca di Trebisonda, siamo indotti a pensare che Alessio I abbia in quell'occasione applicato alla lettera la regola aurea per un sovrano che desideri liberarsi di un personaggio scomodo come Gabras, ma troppo influente per essere eliminato: promoveatur ut amoveatur.

Questa scelta dovette tenere in considerazione che negli anni Ottanta dell'XI secolo il governo centrale aveva oramai perso il controllo sulla Chaldia, allora una sorta di enclave romea nell'Anatolia selgiuchide: rendere perciò ufficiale il potere che Teodoro colà già amministrava di fatto, non avrebbe potuto compromettere l'integrità della basileia o fomentare una ribellione. In primo luogo perché Gabras, quantunque avesse conquistato Trebisonda dai Turchi, aveva agito senza alcun mandato imperiale, né tantomeno aveva compiuto un atto di sottomissione al basileus dopo essere riuscito nell'impresa e quindi, in teoria, era ancora un ribelle. Nella peggiore delle ipotesi avrebbe continuato a governare in modo indipendente come prima della sua visita costantinopolitana. In seconda istanza probabilmente Alessio I immaginava che Teodoro fosse il solo ad avere il polso della situazione sul Ponto e di conseguenza l'unico in grado di difendere quell'estrema provincia bizantina e magari di strappare ai Turchi qualche altro lembo di terra. Poco importava l'eventualità che non rispondesse ai comandi dell'imperatore e decidesse autonomamente, dato che in quel momento Alessio I non avrebbe potuto incidere granché sulle sorti della lontana e marginale Chaldia. In ogni caso il Comneno era pienamente consapevole dell'azzardo che la nomina di Gabras comportava e per questa ragione si era cautelato tenendo in ostaggio Gregorio, in modo tale da 
evitare che l'autonomia del duca di Trebisonda degenerasse in aperta ostilità. Benché questa operazione fosse stata tutto sommato efficace nel limitarne l'esuberanza, evidenziò in maniera inequivocabile che la relazione tra l'imperatore e Teodoro non era quella tra un sovrano e un suo funzionario, bensì tra due sovrani, seppure di diversa natura. Gabras riconosceva la supremazia di Alessio I e accettava il ruolo di cliente, ma contemporaneamente Alessio I non aveva altro modo per garantirsi la fedeltà di Gabras se non attraverso la minaccia per le sorti di suo figlio. Lo strumento dell'ostaggio non era affatto una novità nelle strategie politiche romane e bizantine. Tale strumento costituiva infatti la prassi sia per i sovrani tardoantichi che desideravano educare alla romanità i rampolli delle stirpi principesche barbariche e garantirsene l'amicizia nel momento in cui avessero assunto la guida del loro popolo, sia per i regnanti medievali il cui intento fosse di includere nella famiglia imperiale i potentati ai confini della basileia [Nečaeva 2014, 54-56; Chrysos 1992, 25-39; Shepard 1992, 41-71]. Benché si trattasse di una pratica consolidata nelle relazioni internazionali, era tuttavia alquanto inusuale in una circostanza come quella della nomina di Teodoro a duca di Trebisonda, il quale era formalmente un ufficiale bizantino incaricato del governo di una provincia imperiale. In sostanza, tenendone in ostaggio il figlio, Alessio I riconosceva implicitamente l'autonomia del governo di Teodoro.

Un'ulteriore plastica manifestazione dell'ambiguità intrinseca nei rapporti tra Alessio I e Teodoro è rappresentata dall'istituzione di una zecca a Trebisonda intorno agli anni Ottanta dell'XI secolo, dunque in un periodo compreso tra la riconquista della città da parte di Gabras e i primi anni seguiti al suo rientro da Costantinopoli in veste di duca. Tenuto conto della cronologia e della qualità della monetazione, è ragionevole supporre che la decisione in merito alla costituzione della zecca sia stata presa in maniera autonoma da Teodoro. In effetti, se da un lato il conio, la forma e il peso irregolare delle monete testimoniano un'officina sganciata dal circuito delle zecche imperiali, il perdurare 
dell'isolamento in cui la Chaldia versava in quegli anni e il conseguente diradarsi dei contatti con il resto della Romania, lascia presumere che forse la circolazione monetaria avesse subito un calo sensibile e reso necessaria una produzione per l'uso locale ${ }^{56}$. Se la creazione di una zecca apparentemente 'indipendente' sembra configurare un'esibizione di autonomia politica, l'iconografia e l'epigrafia della monetazione di Gabras contraddicono in parte questo asserto. Benché le immagini dei folleis ${ }^{57}$ trebisontini presentino una conformazione abbastanza inusuale nell'ambito della numismatica bizantina, in esse il richiamo all'autorità imperiale è lampante. Nella maggioranza dei folleis campeggiano l'effige di Alessio I o il suo nome senza che l'autonomia politica di Teodoro trapeli in alcun modo, a eccezione di due piccoli gruppi monetali dove appaiono i monogrammi di san Teodoro stratelata ${ }^{58}$, omonimo di Gabras, o quello di sant'Eugenio ${ }^{59}$, patrono di Trebisonda e Paipert. Nonostante la palese condizione di signore territoriale semi-indipendente, cui peraltro Alessio I acquiesceva, autorizzasse Teodoro a intraprendere iniziative senza il preventivo avallo del governo centrale, il prestigio del quale ancora godeva la figura del sovrano impose al dinasta pontico di riconoscerne la supremazia, conferendo a un tempo validità alle monete, la sola garanzia di autenticità delle quali risiedeva proprio nel basileus.

\footnotetext{
${ }^{56}$ Come attesta la circolazione quasi esclusivamente limitata al Ponto delle monete di produzione trebisontina e la prosecuzione dell'attività dell'officina anche in età 'post-Gabras', con i folleis di Isacco II Angelo (1185-1195, 1203): Bellinger, Grierson, Hendy, IV/1, 427-434; Bendall 1977, 126-136; 1979, 213-217; 1982, 163.

${ }_{57}$ In quel periodo le monete di rame standard nell'Impero: Grierson 1999, 17-22.

${ }_{58}$ Come nel sigillo personale di Teodoro Gabras: Bryer, Dunn, Nesbitt 2003, 6465 ; e dunque riconducibili con una certa sicurezza al dinasta pontico. Per le monete: Bellinger, Grierson, Hendy 1966-1999, IV/1, 431-432.

${ }_{59}$ Per questo gruppo, sovraconiato sui follis con l'effige o il nome del basileus Bendall 1982, 163; prima e Hendy, in Bellinger, Grierson, Hendy, IV/1, 434; poi hanno stimato una datazione tra il 1080 e il 1110.
} 
Abbiamo osservato come attorno a Teodoro Gabras siano fiorite nel corso del medioevo alcune tradizioni locali, poi condensatesi tra la metà del Settecento e la fine dell'Ottocento nel Sinassario e nella Ballata confezionata da Antimo Gabras. Una resistenza così prolungata nel tempo e ben oltre le soglie del medioevo del ricordo delle imprese di Teodoro attesta il radicamento nell'immaginario collettivo dei Cristiani del Ponto della sua figura come eroe locale nella difesa dall'invasione turca. Un radicamento che, tuttavia, non era proprio solo della cultura greco-pontica, ma fu in larga parte condiviso dalla tradizione letteraria turcomanna del Melikdanishmendname. Quest'ultimo è il romanzo epico, la cui sola versione esistente risale circa al 1360 ${ }^{60}$, che raccoglie le imprese degli emiri turcomanni danishmendidi, i vicini e principali avversari di Teodoro Gabras, che si erano insediati in una data imprecisata tra la seconda metà degli anni Settanta e la prima metà degli anni Ottanta dell'XI secolo nel Djanik, un'area che comprendeva parte del litorale dell'Eusino a Oriente della Chaldia e alcuni territori a sud delle Alpi Pontiche, e contendevano insieme al dinasta pontico la supremazia sull'Anatolia nord-orientale ai sultani selgiuchidi [Cahen 1968, 81-84; Oikonomides 1992, 189-207]. Proprio perché non si tratta di una fonte storica vera e propria, l'epopea consente di cogliere meglio quale considerazione avessero di Teodoro le popolazioni di etnia turca e turcomanna. Nel Melikdanishmendname Gabras può essere individuato in una componente dell'eroe cristiano Shah-i-Shattat ${ }^{61}$, una figura composta di diversi individui storici e non, che fu uno degli antagonisti dell'emiro Danishmend Ghazi (?-1104), ed è sempre Teodoro, insieme ai suoi familiari, a nascondersi dietro a uno dei personaggi immaginari di Metropid e dei suoi figli Nikola, Kosta, Gavras e Mihail, difensori delle popolazioni cristiane del Ponto ${ }^{62}$. In entrambe le versioni

60 Melikoff 1960, I, 133.

${ }_{61}$ Ibidem, I, 203-204, 214-222, 435-436.

62 Ibidem, I, 157-159, 198-199, 423-452. 
Gabras appare come il campione dei Cristiani contro le scorrerie di Danishmend Ghazi, il solo ostacolo che si frapponga all'invasione turcomanna della Chaldia e delle regioni circostanti. Ciò che più salta all'occhio è l'assenza dallo scenario descritto nel racconto delle gesta dei Danishmendidi delle principali forze che si contendevano in quegli anni il controllo dell'Anatolia, ossia Bisanzio e il sultanato selgiuchide. Il Melikdanishmendname riproduce, infatti, una dialettica squisitamente locale, dove non trovano spazio né l'imperatore di Costantinopoli, né gli eserciti manovrati dal sultano di Rûm, e in un certo senso conferma il dato storico della frammentazione politica estrema dell'Anatolia di fine XI secolo e dell'incapacità bizantina e selgiuchide di esercitare un controllo effettivo sui signori locali che pure erano loro formalmente dipendenti. Mentre l'impero si disinteressava dei destini del Ponto, Teodoro Gabras era l'unica autorità presente in loco che si ergesse contro i Turchi nella Chaldia del post-Manzicerta, di conseguenza l'unica che i Romei riconoscessero e che, dunque, potesse sopravvivere nella tradizione greca e in quella turcomanna ${ }^{63}$.

\section{Un bilancio}

Il quadro che emerge dall'analisi delle fonti che concernono Teodoro Gabras è quello di un esponente tipico della classe arcontale pontica capace di inserirsi nel vuoto di potere della Chaldia successivo all'occupazione turca di Trebisonda e di sfruttare le prassi politiche proprie dei ceti dirigenti locali per assicurarsi il consenso delle élite guerriere del Ponto e mantenere così il controllo della regione. Sebbene fosse inserito nel sistema di alleanze dinastiche elaborato dagli imperatori comneni, Teodoro era fuori dal diretto controllo di Costantinopoli, giacché

\footnotetext{
63 Per la prosopografia dei Gabras che si celano dietro ai personaggi del Melikdanishmendname: Bryer 1980, 178-179.
} 
la fonte del suo potere e delle sue ricchezze non risiedeva nel basileus, quanto piuttosto nelle prerogative che aveva acquisito e poi conservato in seguito alla conquista della metropoli pontica e del suo circondario grazie alle sue abilità militari, al suo radicamento economico e alla rete delle sue amicizie nella regione e all'incapacità del sovrano di assumere concretamente la direzione del governo locale. L'acquiescenza dell'imperatore alla sua condotta autonoma - in misura significativa dettata anche dalla difficile situazione geopolitica dell'impero all'avvento di Alessio ${ }^{64}$ - evidenziata dalla nomina a duca di Trebisonda in contemporanea alla presa in ostaggio di Gregorio Gabras, non soltanto sottolineava l'impotenza del governo centrale nell'intervenire sulle vicende della Chaldia, ma forniva un segnale in merito al sorgere di una specie di 'microcosmo pontico' alimentato sia dall'isolamento geografico, sia dalla semi-indipendenza di Teodoro. Dopo gli episodi che seguirono la disfatta di Romano IV era improbabile che la Chaldia di fine XI secolo potesse essere governata attraverso gli strumenti canonici dell'amministrazione bizantina ${ }^{65}$. Alessio I, perciò, fu costretto ad acconsentire al rientro di Gabras, benché fosse conscio che in questo modo non avrebbe incrementato la propria popolarità, né riaffermato la propria autorità a Trebisonda, ma soltanto ammantato dei crismi della legalità il potere de facto del duca, al quale i Pontici avrebbero continuato in ogni caso a rivolgersi come unico referente per le questioni concernenti il presidio del territorio. La conquista di Trebisonda e di Paipert e il martirio avevano concesso imperitura fama di campione dei cristiani della Chaldia e un'aura di santità a Teodoro e in ragione di ciò un posto di rilievo nella cultura popolare e nella letteratura epica. La natura delle relazioni tra la Chaldia e Costantinopoli era mutata in maniera irreversibile dopo

${ }_{64}$ Con i Normanni in Epiro e i Turchi in Bitinia sulla sponda opposta del Bosforo.

${ }_{65}$ Benché l'ossatura fosse probabilmente sopravvissuta all'occupazione turca, rimanendo pressoché immutata sino all'avvento dell'Impero di Trebisonda, che ancora se ne serviva per il controllo del territorio nel XV secolo: Bryer 1966, 152-160. 
l'esperienza di Teodoro e per tutto il XII secolo la basileia non sarebbe più stata in grado di recuperare il pieno controllo del territorio. A periodi in cui la sovranità imperiale pareva ripristinata si alternarono improvvisi ritorni delle istanze separatiste pontiche, fino a quando, grosso modo in contemporanea con la caduta della Polis in mano ai crociati, Alessio e Davide Comneni entrarono a Trebisonda con le truppe della regina Tamara di Georgia [Savvides 2009, 44; Fasolio 2015, 212] e la separazione della Chaldia dal resto di Bisanzio fu definitiva. Con il trascorrere dei secoli, allorché l'indipendenza politica del Ponto dal resto della Romania si era consolidata e il suo particolarismo sociale e culturale era divenuto chiaramente percepibile dai contemporanei, nella memoria collettiva dei Romei e dei Turchi del luogo Teodoro Gabras fu avvertito sempre più come un precursore della realtà loro coeva, e come tale rispettato e venerato.

\section{Bibliografia}

\section{Fonti primarie}

Anne Comnène 1937-1945, Alexiade, 3 voll., ed. Leib B., Paris: Les belles lettres.

Costantino Porfirogenito 1952, De thematibus, ed. Pertusi A., Città del Vaticano: Biblioteca Apostolica Vaticana (Studi e Testi», CLX).

Dostourian A. E. (trad.) 1993, Armenia and the Crusades: Tenth to Twelfth Centuries. The Chronicle of Matthew of Edessa, Lanham-London: University Press of America.

Étienne Asołik de Tarôn 1917, Histoire Universelle, trad. Macler F., Paris: Ernest Leroux (Publications de l'école de langues orientales vivantes, série I, tome XVIII bis).

Ioannes Scylitzes 1973, Synopsis historiarum, ed. Thurn H., Berolini-Novi eboraci: de Gruyter (Corpus Fontium Historiae Byzantinae, V, Series Berolinensis).

Ioannes Zonaras 1841-1897, Epitomae historiarum libri XVIII, 3 voll., eds. Pinder M., Büttner-Wobst T., Bonnae: Impensis Ed. Weberi (Corpus Scriprorum Historiae Byzantinae, XLVII-XLIX).

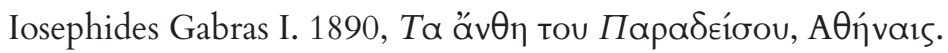


Kratchovski I., Vasiliev A. 1936, Histoire de Yahyā Ibn-Sa`̀d d'Antioche continuateur de Sa`̃d-Ibn-Bitrīq, in Patrologia Orientalis, XXIII/3, Paris: Firmin-Didot, 343-519.

Leo Diaconus Caloënsis 1828, Historiae libri X, ed. Hase C. B., Bonnae: Impensis Ed. Weberi (Corpus Scriprorum Historiae Byzantinae, XXX).

Mélikoff I. (ed.) 1960, Dānişmendnāme. La Geste de Melik Dānişmend, 2 voll., Paris: A. Maisonneuve (Bibliothèque archéologique et historique de l'Institut français d'archéologie d'Istanbul, 10-11).

Michel le Syrien 1899-1910, Chronique, 4 voll., ed. Chabot J.-B., Paris: Ernest Leroux.

Miguel Ataliates 2002, Historia, ed. Perez Martin I., Madrid: Consejo superior de investigaciones cientificas (Nueva Roma, 15).

Nicéphore Bryennios 1975, Histoire, ed. Gautier P., Bruxelles: Byzantion (Corpus Fontium Historiae Byzantinae, IX, Series Bruxellensis).

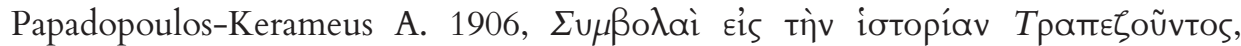
«Византийский Временник», XII: 132-147.

Rosenqvist J. O. (ed.) 1996, The Hagiographic Dossier of St. Eugenios of Trebizond in Codex Athous Dionisiou 154, Uppsala: Almqvist \& Wiksell (Acta Universitatis Upsaliensis. Studia Byzantina Upsaliensia, 5).

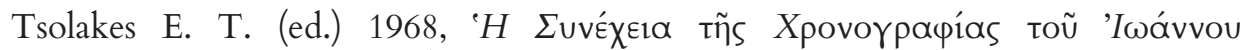

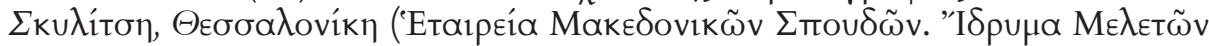
Xeporovíoou той “Aıноч, 105).

Yahyā al-Anțakī 1998, Cronache dell'Egitto fătimide e dell'impero bizantino (937-1033), ed. Pirone B., Milano: Jaca book (Patrimonio Culturale Arabo Cristiano, 3).

Zakythinos D. 1932, La chrysobulle d'Alexis III Comnène empereur de Trébizonde en faveur des Vénitiens, Paris: Les belles lettres (Collection de l'Institut néo-hellénique de l'Université de Paris, 12).

\section{Fonti secondarie}

Andriollo L. 2017, Constantinople et le provinces d'Asie Mineure, IXe -XIe siècle. Administration impériale, sociétés locales et rôle de l'aristocratie, Leuven-Paris-Bristol: Peeters (Travaux et mémoires du Centre de recherche d'histoire et civilisation de Byzance. Monographies, 52).

Andriollo L., Métivier S. 2017, Quel rôle pour les provinces dans la domination aristocratique de XIe siècle?, "Travaux et Mémoires», 21: 505-530.

Angold M. 1997, The Byzantine Empire, 1025-1204: a political history, London-New York: Longman (ed. or. 1984).

Bartikian H. 1996, Les Gaurades à travers les sources arméniennes, in Garsoïan N. (ed.), L'Arménie et Byzance. Histoire et Culture, Paris: Publications de la Sorbonne (Byzantina Sorboniensia, 12), 19-30. 
Bausani A. 1965, s.v. "Gabr», in B. Lewis et al. (eds.) The Encyclopedia of Islam2, II, London-Leiden: E. J. Brill, 970.

Bellinger A. R., Grierson P., Hendy M. (eds.) 1966-1999, Catalogue of the Byzantine Coins in the Dumbarton Oaks Collection and in the Whittemore Collection, 5 voll., Washington D. C.: Dumbarton Oaks Research Library and Collection.

Bendall S. 1977, The Mint of Trebizond under Alexius I and the Gabrades, "The Numismatic Chronicle ${ }^{7}, 17:$ 126-136.

- 1979, The Coinage of Trebizond under Isaac II (A. D. 1185-1195). With a Note on an Unfinished Byzantine Die, "American Numismatic Society Museum Notes», 24: 213-217.

- 1982, A New Twelfth-Century Byzantine Coin from the Mint of Trebizond, "The Numismatic Chronicle», 142: 163.

Beneševič V. N. 1912-1925, Monumenta Sinaitica archaeologica et palaeographica, 2 voll., Petropolis.

Bosworth C. E. 1998, The Ghaznavids, in Asimov M. S., Bosworth C. E. (eds.), History of Civilisations of Central Asia, IV, Paris: Unesco, 103-124.

Bryer A. M. 1966, Rural Society in the Empire of Trebizond, "Apxeíov Пóvtou», 28: 152-160.

- 1980, A Byzantine Family: the Gabrades, c. 979-c. 1653, in Id., The Empire of Trebizond and the Pontos, London: Variorum Reprints (ed. or. 1970, «University of Birmingham Historical Journal», XII), 164-187.

Bryer A. M., Dunn A., Nesbitt J. W. 2003, Theodore Gabras, Duke of Chaldia (†1098) and the Gabrades: Portraits, Sites and Seals, in Laiou A. E., Avramea A., Chrysos

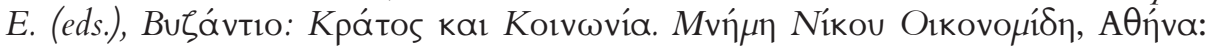

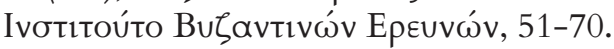

Bryer A. M., Fassoulakis S., Nicol D. M. 1980, A Byzantine Family: the Gabrades. An Additional Note, in A. M. Bryer, The Empire of Trebizond and the Pontos, London: Variorum Reprints (ed. or. 1975, «Byzantinoslavica», XXXVI), 38-45.

Bryer A. M., Winfield D. 1985, The Byzantine monuments and topography of the Pontos, 2 voll., Washington D. C.: Dumbarton Oaks Research Library and Collection (Dumbarton Oaks Studies, XX).

Cahen C. 1968, Pre-Ottoman Turkey: a General Survey of the Material and Spiritual Culture and History, c. 1071-1330, New York: Taplinger.

Cheynet J.-C. 1980, Mantzikert: un désastre militaire?, «Byzantion», L: 410-418.

- 1983, Dévaluation des dignités et dévaluation monétaire dans la seconde moitié du XIe siècle, «Byzantion», LIII: 453-477.

- 1984, Toparque et topotèrètès à la fin du XIe siècle, "Revue des études byzantines", 42: 215-224. 
- 1996, Les Arméniens de l'empire en Orient de Costantin X à Alexis Comnène (10591081), in Garsoïan N. (ed.), L'Arménie et Byzance. Histoire et Culture, Paris: Publications de la Sorbonne (Byzantina Sorboniensia, 12), 67-78.

- 1998, La résistance aux turcs en Asie Mineure entre Mantzikert et la première croisade, in Balard M. et al. (eds.) EYYXIA: mélanges offerts à Hélène Ahrweiler, 2 voll., Paris: Publications de la Sorbonne (Byzantina Sorboniensia, 16), I, 131-147.

- 2008, Les Brachamioi, in Id., La societé byzantine. L'apport des sceaux, 2 voll., Paris: Association des amis du Centre d'histoire et civilisation de Byzance (Bilans de recherche, 3), II, 377-412.

Cheynet J.-C., Vannier J.-F. 1986, Études prosopographiques, Paris: Publications de la Sorbonne (Byzantina Sorboniensia, 5), 57-74.

Chrysos E. 1992, Byzantine diplomacy, A. D., 300-800: means and ends, in Shepard J., Franklin S. (eds.), Byzantine diplomacy, Aldershot: Variorum (Papers from the Twenty-Fourth Spring Symposium of Byzantine Studies, Cambridge, March 1990), 25-39.

Darrouzes J. 1977, Questions de droit matrimonial: 1172-1175, «Revue des études byzantines", 35: 107-157.

Fasolio M. 2015, Una comparazione possibile? La crisi di Bisanzio e lo sviluppo dei principati separatisti di Trebisonda e d'Epiro, in Luongo A., Paperini M. (eds.), Medioevo in formazione. Studi storici e multidisciplinarità, Livorno: Debatte (Confronti, 8), 210221.

Glykatzi-Ahrweiler H. 1976, Recherches sur la société byzantine au XIe siècle: nouvelles hiérarchies et nouvelles solidarités, «Travaux et Mémoires», 6: 99-124.

Grierson P. 1999, Byzantine Coinage, Washington D. C.: Dumbarton Oaks Research Library and Collection.

Guilland R. 1967, Recherches sur les institutions byzantines, 2 voll., Amsterdam: Adolf M. Hakkert.

Haldon J., L'armée au XIe siècle. Quelques questions et quelques problèmes, «Travaux et Mémoires», 21: 581-593.

Hendy M. F. 1985, Studies in the Byzantine Monetary Economy, c. 300-1450, Cambridge: Cambridge University Press.

Holmes C. 2005, Basil II and the Governance of the Empire, Oxford: Oxford University Press.

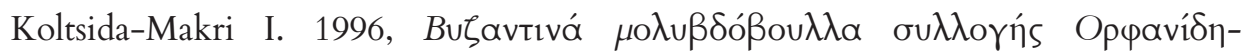

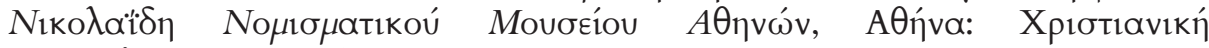

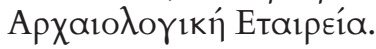

Laiou A. E. 2002, Exchange and Trade, Seventh-Twelfth Centuries, in Id. (ed.), The Economic History of Byzantium: from the Seventh through the Fifteenth Century, 3 voll., 
Washington D. C.: Dumbarton Oaks Research Library and Collection (Dumbarton Oaks Studies, 39), 697-770.

Laiou A. E., Morrisson C. 2007, The Byzantine economy, Cambridge: Cambridge University Press.

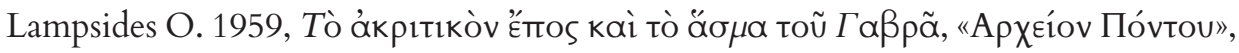
23: 33-38.

Likhachyov N. P. 1911, Историческое значение итало-греческой иконописи. Изображения Богоматери в произведениях итало-греческих иконописцев и их влияние на композиции некоторых православныхрусских икон, Санкт-Петербург: Г. Лисснера и Д. Собко.

Magdalino P. 2009, Court Society and Aristocracy, in Haldon J. (ed.), A Social History of Byzantium, Malden: Wiley-Blackwell, 212-232.

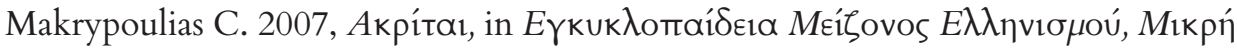
Aớa, http://www.ehw.gr/l.aspx?id=3478.

Moravcsik G. 1984, Byzantinoturcica, 2 voll., Berlin-Leiden: E. J. Brill (Berliner Byzantinischer Arbeiten, 10; ed. or. Budapest 1942-1942).

Morrisson C. 1976, La dévaluation de la monnaie byzantine au XIe siècle: essai d'interprétation, «Travaux et Mémoires», 6: 3-48.

Mutafciev P. 1932, Bulgares et Roumains dans l'histoire des pays danubiens, Sofia: Danov.

Nečaeva E. 2014, Embassies - Negotiations - Gifts: Systems of East Roman Diplomacy in Late Antiquity, Stuttgart: Franz Steiner Verlag (Geographica Historica, 30).

Neville L. 2004, Authority in Byzantine provincial society, 950-1100, Cambridge: Cambridge University Press.

Oikonomides N. 1976, L'évolution de l'organisation administrative de l'Empire byzantin au XIe siècle (1025-1118), «Travaux et Mémoires», 6: 125-152.

- 1992, Les Danishmendides, entre Byzance, Bagdad et le sultanat d'Iconium, in Id., Byzantium from the ninth century to the fourth crusade. Studies, texts, monuments, Aldershot (ed. or. 1983, «Revue numismatique ${ }^{6}$, 25: 189-207), 189-207.

Oikonomides N., Nesbitt J., Mc Geer E. (eds.) 1991-2009, Catalogue of Byzantine Seals at Dumbarton Oaks and at the Fogg Museum of Art, 6 voll., Washington D.C.: Dumbarton Oaks Research Library and Collection.

Pirivatrić S. 1997, Самуилова држава. Обим и карактер, Београд: Виэантинолошки институт САНУ (Византолошки Институт Спрске Академије Наука и Уметности, 21).

Prokić B. 1906, Die Zusätze in der Handschrift des Johannes Skylitzes, codex Vindobonensis hist. graec. LXXIV. Ein Beitrag zur Geschichte des sogenannten Westbulgarischen Reiches, München: H. Kutzner. 


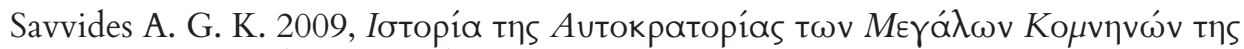

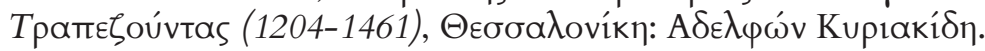

Schlumberger G. L. 1894, Sceaux byzantins inédites (3e série), "Revue des Études Grecques», VII (27): 319-336.

Seibt W., Zarnitz M.-L. 1997, Das byzantinische Bleisiegel als Kunstwerk: Katalog Zur Ausstellung, Wien: Verlag der Österreichischen Akademie der Wissenschaften.

Shepard J. 1992, Byzantine diplomacy, A. D., 800-1204: means and ends, in Shepard J., Franklin S. (eds.), Byzantine diplomacy, Aldershot: Variorum (Papers from the Twenty-Fourth Spring Symposium of Byzantine Studies, Cambridge, March 1990), 41-71.

Skoulatos B. 1980, Les personnages byzantines de l'Alexiade: analyse prosopographique et synthèse, Louvain: Nauwelaerts (Universitè de Louvain. Recueil de travaux d'histoire et de philologie, série 6, fasc. 20).

Vannier J.-F. 1998, Notes généalogiques byzantino-géorgiennes, Balard M. et al. (eds.) EYUXIA: mélanges offerts à Hélène Ahrweiler, 2 voll., Paris: Publications de la Sorbonne (Byzantina Sorboniensia, 16), II, 673-688.

Vryonis jr. S. 1971, The Decline of Medieval Hellenism in Asia Minor and the Process of Islamization from the Eleventh through the Fifteenth Century, Berkley-Los AngelesLondon: University of California Press (Publications of the Center for Medieval and Renaissance Studies, IV).

Yarnley C. J. 1972, Philaretos: Armenian Bandit or Byzantine General, "Revue des études arméniennes», 9: 331-353. 